\title{
NeuroRegulation
}

\section{Neurofeedback for Chemotherapy Induced Neuropathic Symptoms: A Case Study}

\author{
Sarah Prinsloo ${ }^{1 *}$, Stephanie Gabel ${ }^{2}$, Lauren Data ${ }^{3}$, Randall Lyle ${ }^{4}$ \\ ${ }^{1}$ Private Practice, Houston, Texas, USA \\ ${ }^{2}$ St. Mary's University, San Antonio, Texas, USA \\ ${ }^{3}$ University of Houston, Clear Lake, Texas, USA \\ ${ }^{4}$ Marriage and Family Therapy Program, Mt Mercy University, Cedar Rapids, lowa, USA
}

*Address correspondence to: Sarah Prinsloo, PhD, 21340 Provincial Blvd, Katy, TX 77450. Email:

SarahA Prinsloo@hotmail.com

Copyright: ( 2014 Prinsloo et al. This is an Open Access article distributed under the terms of the Creative Commons Attribution License (CC-BY).

\begin{abstract}
This case report highlights qEEG and LORETA measures as well as training protocols for a client experiencing self-reported chemotherapy-induced peripheral neuropathy (CIPN). Specifically, we were interested in whether or not the presentation of visual changes secondary to chemotherapy would be responsive to neurofeedback training (NFB). After 20 sessions of NFB, the client reported improvement in all reported symptoms of neuropathy, although her vision did not improve as drastically as the other symptoms.
\end{abstract}

Keywords: neuropathy, neurofeedback, LORETA, qEEG

\section{Introduction}

This is a case study of a 60-year-old woman who was approximately two years post diagnosis of breast cancer and who suffered from neuropathic symptoms after chemotherapy. Neuropathy is a common consequence of cancer and its treatments, where sensory nerves, motor nerves, and autonomic nerves can be affected, such that daily activities such as driving a car, putting on clothing, using utensils, and walking can become difficult if not impossible. In addition to the more common symptom complaints listed above, patients may also experience other symptoms such as visual disturbances that limit their ability to perform daily activities. Neuropathic symptoms may not only impact quality of life but may also affect patient outcomes because of resulting treatment delays, dose reductions, and treatment discontinuation (Vitacco, Brandeis, Pascual-Marqui, \& Martin, 2002). The incidence of chemotherapy-induced peripheral neuropathy (CIPN) is estimated to be as much as $71 \%$, depending on the class of agents used (Taxotere, 2014; Abraxane, 2014; Navelbine, 2014). 
Of the complaints patients may have of neuropathic symptoms, chemotherapy-related ocular toxicities are underreported as the medical team prioritizes cancer treatment (Singh \& Singh, 2012). Complaints of visual side effects range from blurred vision to decreased visual acuity, optic neuropathy, and rarely produced total loss of vision at the early stages (Caraceni, Martini, Spatti, Thomas, \& Onofrj, 1997; Singh \& Singh, 2012). Cases of visual disturbances from platinum (cisplatin and carboplatin) chemotherapeutic agents are more prevalent than visual disturbances secondary to paclitaxel; however, previous cases of transient visual disturbance during paclitaxel infusions were reported to affect the retina and optic nerve pathways (Scaioli et al., 2006; Capri et al., 1994).

In most cases, visual changes secondary to paclitaxel typically show signs of improvement or reach stabilization at the end of chemotherapy treatment (van den Bent et al., 1997). One case report linked a patient's loss of vision to optic nerve damage during infusion of the taxane drug, docetaxel. This damage was reversed with high-dose steroid therapy (Moloney et al., 2014); however, not all visual changes after chemotherapy are reversible. Another study reported optic neuropathy and retinopathy in 5 patients with permanent visual damage (Khawly et al., 1996). Therefore visually related signs and symptoms secondary to chemotherapy (such as retinopathy) may be generally reversible, whereas those associated with optic neuropathy often may be permanent (Khawly, Rubin, Petros, Peters, \& Jaffe, 1996). Finally, although paclitaxel as a therapeutic agent is efficacious in promoting apoptosis and the proliferation of cancer cells, as the case reports suggest, neurological symptoms affecting the visual field may also lead to discontinuation of anti-tumor remedies (Lee \& Swain, 2006), hence the severity of the problem and the need for effective treatment alternatives.

It has been found that the direct causes of CIPN include the presence of inflammation and the loss of nerve fibers in the affected area (Gannon, 1971). However, the mechanisms of pain and other neuropathic symptoms, incorporating peripheral receptors, pain pathways, and cortical and subcortical centers where symptoms are perceived, have brought emphasis to the importance of the corticalization of symptoms (Jensen, Greirson, Tracy-Smith, Bacigalupi, \& Othmer, 2007). Since the brain has considerable neuroplastic capabilities, neurofeedback may be of benefit to neuropathy patients presenting with a variety of symptoms.

\section{Background}

At the time of presentation, the client was a 60-year-old, Caucasian female who reported symptoms of peripheral neuropathic symptoms secondary to chemotherapy treatment with paclitaxel. The client started chemotherapy in June and noted neuropathic symptoms started immediately after her first treatment. Her presenting symptoms were that she dropped objects, had total body cramps, stumbled over objects such as chairs, felt like she didn't know where her feet were, had trouble dressing herself and putting on jewelry, had trouble writing, and she could not cut up her food. She also reported changes in her vision such that she didn't feel safe to drive and had difficulty reading road signs, and even found it extremely difficult to cut her own nails. The patient described the visual perceptual problems as "not being able to process an entire scene" and this led to an increased anxiety around driving or going to the store. The patient sought out acupuncture for her blurry vision and chemotherapy-induced peripheral neuropathy. She reported that the blurry vision would get better for about 15 minutes, but then would return to the previous blurred state. After completing her acupuncture treatments, the patient consulted with an ophthalmologist. 
Initially, the ophthalmologist believed her vision problems were due to either dry eye or thin cornea. Both artificial tears and Muro drops for corneal health were administered to the patient to no avail. The neuro-ophthalmic evaluation did not reveal a visual problem that explained her distress. An MRI of the brain also revealed no cause of visual impairment. The patient was advised to discontinue driving and was referred to the department of neuropsychology at a local medical institution for further testing.

Neuropsychological testing revealed mild to moderate impairments of cognitive processing speed and below-expected hand strength and motor dexterity. These deficits were attributed to chemotherapy treatment. The neuropsychologist indicated that slower processing speed at moments at which higher processing is needed (e.g., while driving) could be contributing to her experience of anxiety. The neuropsychologist suggested various anxiety-controlling methods. After this consultation, the patient sought neurofeedback to help her manage the symptoms of chemotherapy-induced peripheral neuropathy.

\section{Methods}

The client underwent an initial qEEG, which showed a decrease in alpha power with a concomitant increase in beta power along the sensorimotor strip and into the parietal and occipital lobes, among other findings (Figure 1).
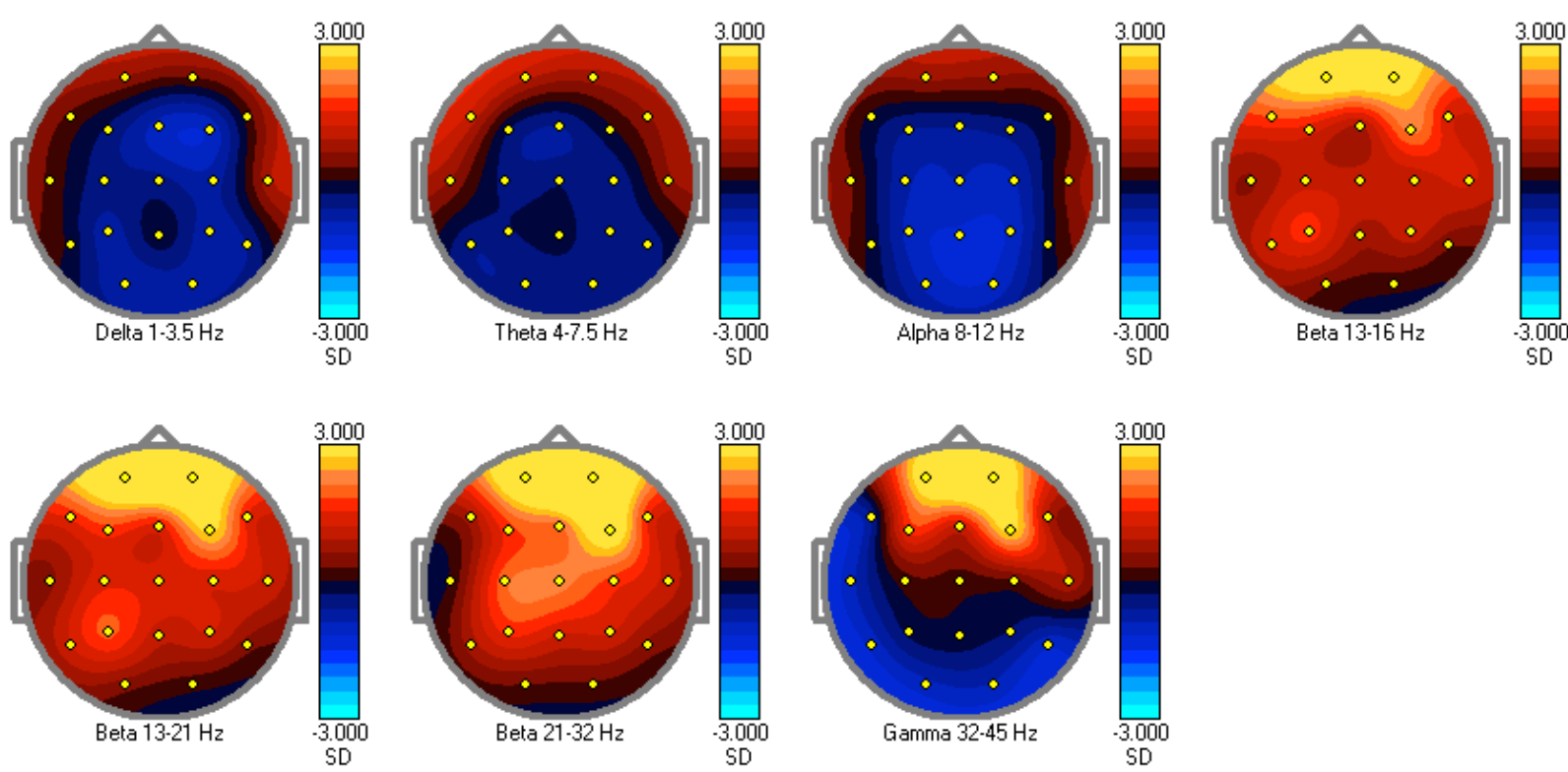

Figure 1. Baseline qEEG (eyes closed).

It was decided to begin neurofeedback with the primary electrode placements at P3, P4 (2channel referential training), with reward frequency set at $8-11 \mathrm{~Hz}$ and inhibit frequencies set at $13-21 \mathrm{~Hz}$ and $4-7 \mathrm{~Hz}$. Prior to her first session, she rated her neuropathic symptoms at a six on a numeric rating scale (NRS), with zero being no symptoms and ten being the worst symptoms imaginable, but reported a decrease in her average symptom rating to a five by session number eight (Figure 2). 


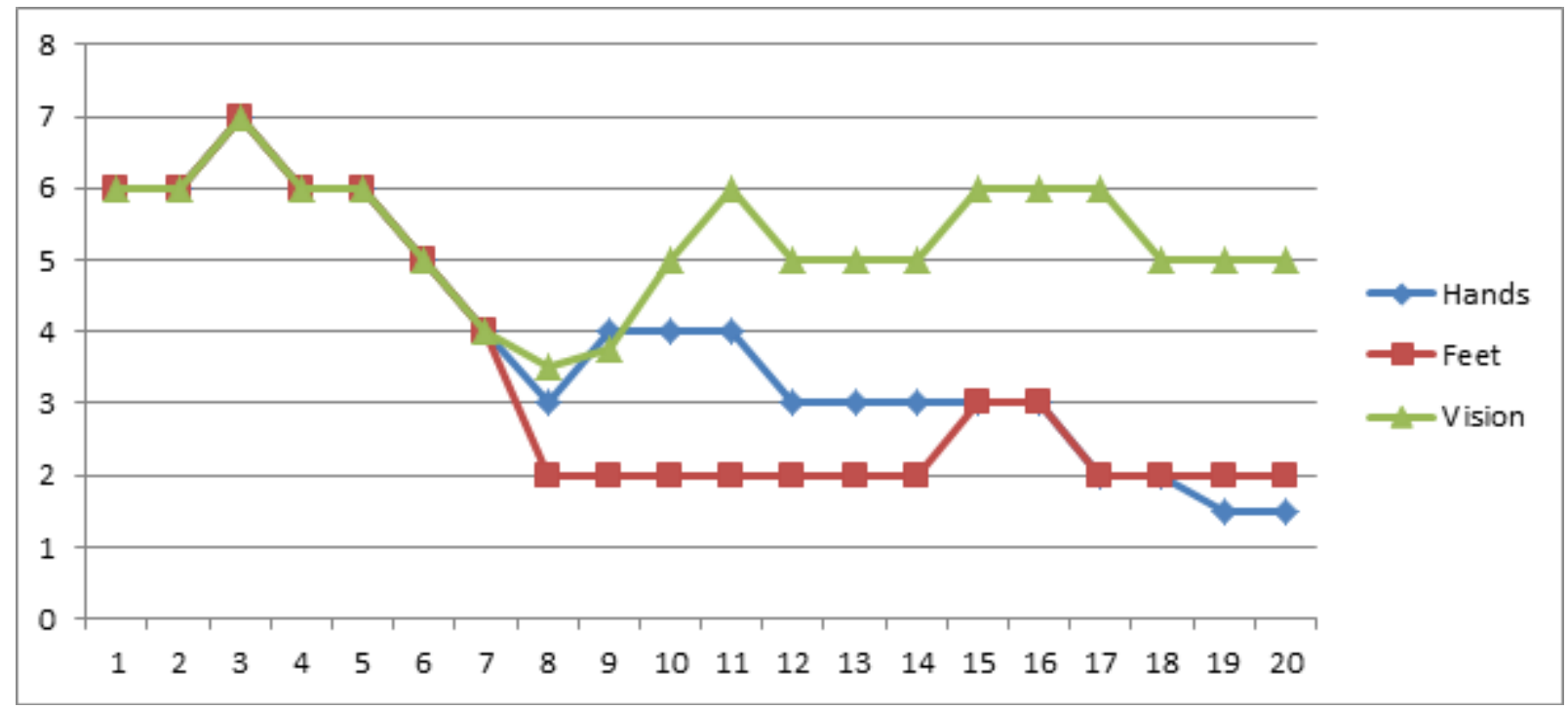

Figure 2. Change in neuropathy scores over 20 sessions of cortical neurofeedback for neuropathic symptoms of hands, feet, and vision.

At that time, we began to ask her to break down her numeric rating scale (NRS) reports to areas she felt were most affected by her symptoms. Her report at session eight was:

$\begin{array}{lll} & \text { Pre } & \text { Post } \\ \text { Hands } & 3 & 3 \\ \text { Feet } & 2 & 2 \\ \text { Vision } & 4 & 3.5\end{array}$

The protocol was changed at session nine to F8, F4, two-channel referential training with reward set at $12-15 \mathrm{~Hz}$ and inhibit frequencies set at 15-21 and $22-36 \mathrm{~Hz}$.

The first session at this new location resulted in the following report:

$\begin{array}{lll} & \text { Pre } & \text { Post } \\ \text { Hands } & 3 & 4 \\ \text { Feet } & 2.5 & 2 \\ \text { Vision } & 4 & 3.75 \text { with transient symptoms of blurriness }\end{array}$

The final protocol change was to T3, T4, two-channel referential with reward set at 13-15 $\mathrm{Hz}$, and inhibits set at $1-3$ and $8-11 \mathrm{~Hz}$. Upon completion of 20 sessions of neurofeedback, her report was as follows:

$\begin{array}{lll} & \text { Pre } & \text { Post } \\ \text { Hands } & 1.5 & 1.5 \\ \text { Feet } & 2 & 2 \\ \text { Vision } & 5 & 5\end{array}$

\section{Results}

At the end of 20 sessions, the client reported that she felt her neuropathy had improved overall since neurofeedback. She reported she sewed a button on for the first time and was able to start picking objects up without dropping them. She had transient visual improvement 
where she could read road signs and objects were less blurry; however, these improvements were not long lasting. Her overall rating of neuropathy was a four, which was a two-point decrease from baseline. From baseline to post-20 session training, we were able to achieve an increase in alpha, which we hypothesize contributed to the client's reported improvement of CIPN (Figure 3).
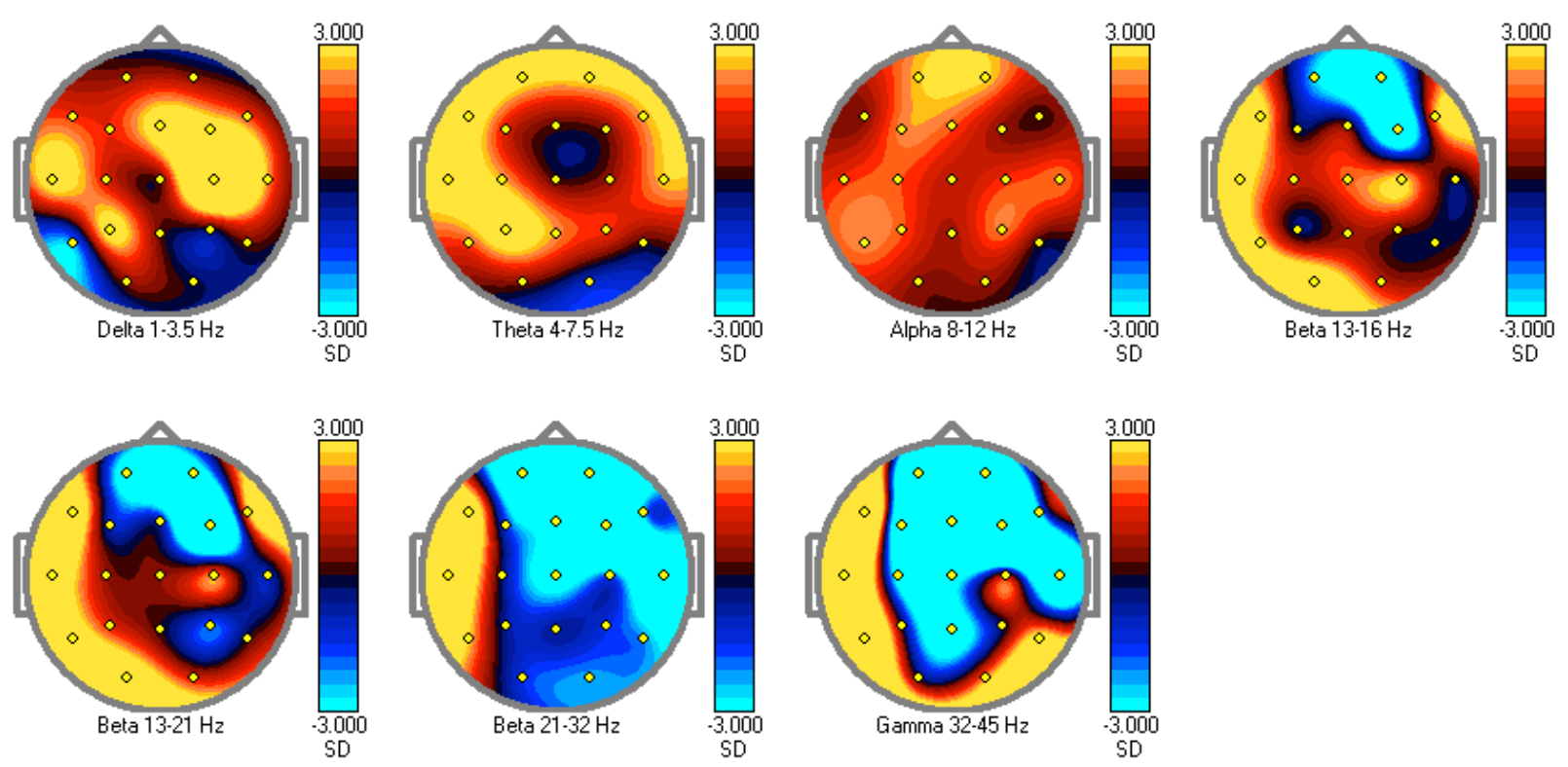

Figure 3. Pre/Post Comparison Maps.

Likewise, LORETA maps demonstrate an increase in alpha activity (Figure 4).

We were also able to assess the client at four months post training. There were no medication changes from post training to the four-month follow up. Her symptom report was as follows:

$\begin{array}{ll}\text { Hands } & 1.5 \\ \text { Feet } & 1 \\ \text { Vision } & 5\end{array}$




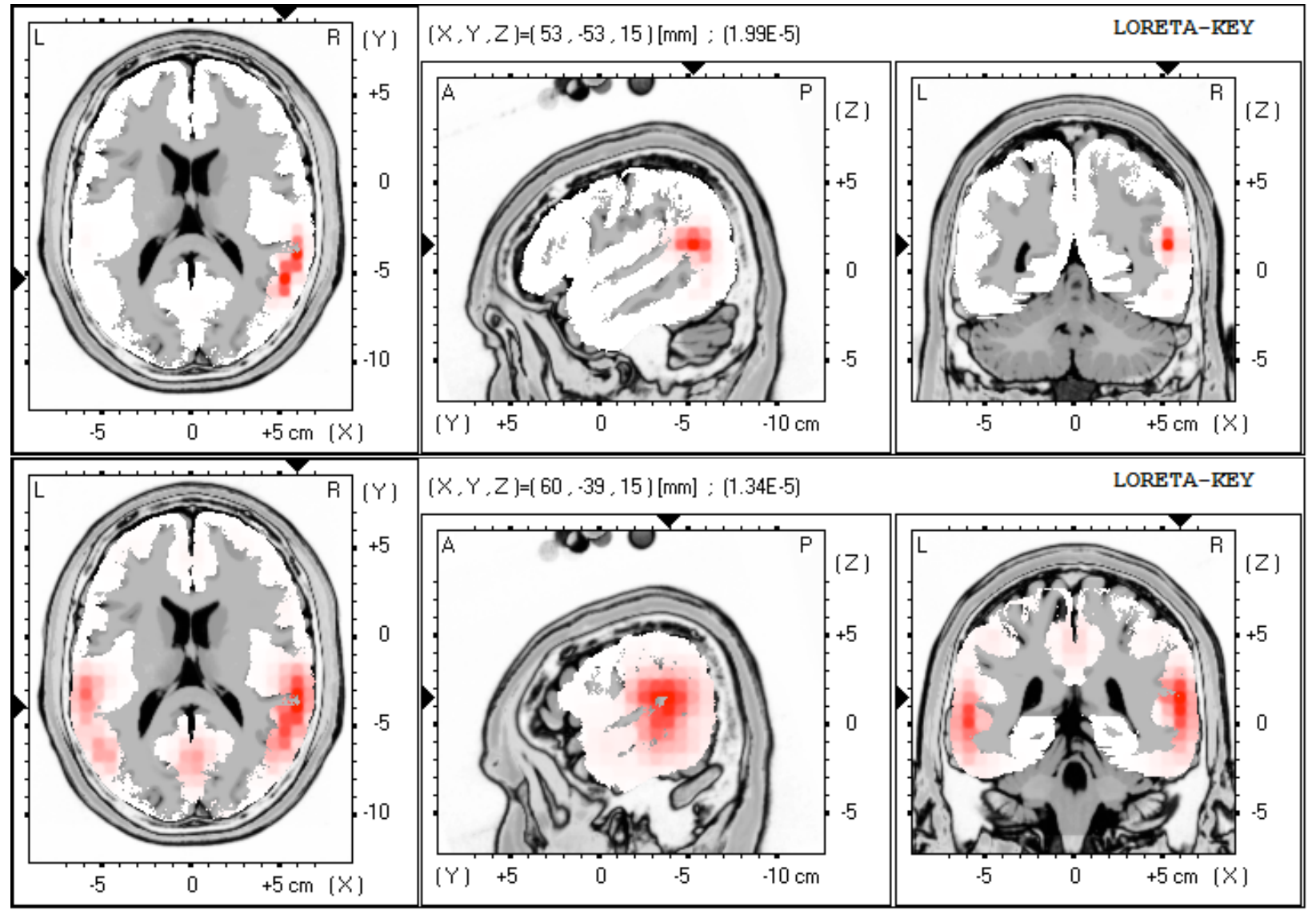

Figure 4. Upper maps: LORETA image for eyes closed alpha $(8-12 \mathrm{~Hz})$ baseline assessment. Lower maps: LORETA image for eyes closed alpha $(8-12 \mathrm{~Hz})$ post 20 sessions.

\section{Discussion}

In conclusion, chemotherapy-induced peripheral neuropathy seems to respond well to cortical neurofeedback, however visual changes secondary to chemotherapy are seemingly not as responsive as neuropathic symptoms in other regions of the body.

Literature suggests that alpha band changes are associated with improvement of visual field detection, improved temporal processing of visual stimuli, detection performance, and visual acuity, specifically with optic neuropathy patients (Sabel et al., 2011). Indeed, this case study suggests that alpha increase after neurofeedback training did in fact correlate with improvement in visual symptom report, even though the training protocols used were decided upon to try to maximize improvement in all symptoms, not solely the client's perception of visual problems. In conclusion, neurofeedback treatment of this patient targeted the full range of neuropathic symptoms and not just those related to vision. However, it was discovered that while somewhat transitory, NFB was also effective with the symptoms related to her vision. It is anticipated that as more and more "baby-boomers" age, we can expect an increase in the types of problems experienced by people receiving chemotherapy. The results of this case offer good evidence that further research into the effectiveness of NFB on CIPN would be of great benefit to many. 


\section{NeuroRegulation}

\section{References}

Abraxane Important Safety Information. (n.d.). Retrieved May 16, 2014, from http://www.abraxane.com/

Becher, R., Schütt, P., Osieka, R., \& Schmidt, C. G. (1980). Peripheral neuropathy and ophthalmologic toxicity after treatment with cisdichlorodiaminoplatinum II. Journal of Cancer Research and Clinical Oncology, 96(2), 219-222.

Capri, G., Munzone, E., Tarenzi, E., Fulfaro, F., Gianni, L., Caraceni, A., ... Scaioli, V. (1994). Optic nerve disturbances: A new form of paclitaxel neurotoxicity. Journal of the National Cancer Institute, 86, 1099-1101.

Caraceni, A., Martini, C., Spatti, G., Thomas, A., \& Onofrj, M. (1997). Recovering optic neuritis during systemic cisplatin and carboplatin chemotherapy. Acta Neurologica Scandinavica, 96, 260-261.

Caro, X. J., \& Winter, E. F. (2011). EEG biofeedback treatment improves certain attention and somatic symptoms in fibromyalgia: a pilot study. Applied Psychophysiology and Biofeedback, 36(3), 193-200. http://dx.doi.org/10.1007/s10484-011-9159-9

Gannon, L. S. R. (1971). Alpha enhancement as a treatment for pain: A case study. Journal of Behavior Therapy and Experimental Psychiatry, 2, 209-13.

Jensen, M., J., Greirson, C., Tracy-Smith, V., Bacigalupi, S., \& Othmer, S. (2007). Neurofeedback for treatment of pain associated with complex regional pain syndrome type I: A case series. Journal of Neurotherapy, 11, 45-53.

Khawly, J. A., Rubin, P., Petros, W., Peters, W. P., \& Jaffe, G. J. (1996). Retinopathy and optic neuropathy in bone marrow transplantation for breast cancer. Ophthalmology, 103(1), 87-95.

Lee, J. J., \& Swain, S. M. (2006). Peripheral neuropathy induced by microtubule-stabilizing agents. Journal of Clinical Oncology, 24(10), 1633-1642. http://dx.doi.org/10.1200/JCO.2005.04.0543

Moloney, T. P., Xu, W., Rallah-Baker, K., Oliviera, N., Woodward, N., \& Farrah, J. J. (2014). Toxic optic neuropathy in the setting of docetaxel chemotherapy: A Case report. BMC Ophthalmology, 14, 18-24. http://dx.doi.org/10.1186/1471-2415-14-18

Navelbine (Vinorelbine Tartrate) Drug information: Indications, dosage and how supplied Prescribing information at RxList. (n.d.). RxList. Retrieved May 16, 2014, from http://www.rxlist.com/navelbine-drug/indications-dosage.htm

Ibric, V. L., \& Dragomirescu, L, G. (2009). Neurofeedback in pain management. In T. H. Budzynski, H. K. Budzynski, J. R. Evans, \& A. Abarbanel (Eds.), Introduction to quantitative EEG and neurofeedback: Advanced theory and applications (2nd ed., pp. 417-450). Boston, MA: Academic Press. 
Ostrow, S., Hahn, D., Wiernik, P. H., \& Richards, R. D. (1978). Ophthalmic toxicity after cisdichlorodiaminoplatinum (II) therapy. Cancer Treatment Reports, 62, 1591-1594.

Sabel, B., Fedorov, A., Naue, N., Borrman, A., Herrmann, C., \& Gall, C. (2011). Non-invasive alternating current stimulation improves vision in optic neuropathy. Restorative Neurology and Neuroscience, 29, 493-505.http://dx.doi.org/10.3233/RNN-2011-0624

Scaioli, V., Caraceni, A., Martini, C., Curzi, S., Capri, G., \& Luca, G. (2006).

Electrophysiological evaluation of visual pathways in paclitaxel-treated patients. Journal of Neurooncology, 77(1), 79-87. http://dx.doi.org/:10.1007/s11060-005-9008-x

Singh, P., \& Singh, A. (2012). Ocular adverse effects of anti-cancer chemotherapy and targeted therapy. Journal of Cancer Therapeutics and Research, 1(5), 1-7. http://www.hoajonline.com/journals/pdf/2049-7962-1-5.pdf

Taxotere (Docetaxel for Injection) Drug information: Indications, dosage and how supplied Prescribing information at RxList. (n.d.). RxList. Retrieved May 16, 2014, from http://www.rxlist.com/taxotere-drug/indications-dosage.htm

van den Bent, M. J., Vivian, J. M., van Raaij-van den Aarssen, V. J. M., Verweij, J., Doorn, P. A. V., \& Seillevis Smitt, P. A. E. (1997). Progression of paclitaxel induced neuropathy following discontinuation of treatment. Muscle \& Nerve, 20, 750-752.

Vallejo, R., Tilley D. M., Vogel L., \& Benyamin R. (2010). The role of glia and the immune system in the development and maintenance of neuropathic pain. Pain Practice, 10(3), 167-84. http://dx.doi.org/10.1111/j.1533-2500.2010.00367.x

Vitacco, D., Brandeis, D., Pascual-Marqui, R., \& Martin, E. (2002). Correspondence of eventrelated potential tomography and functional magnetic resonance imaging during language processing. Human Brain Mapping, 17(1), 4-12. http://dx.doi.org/:10.1002/hbm.10038 